\section{What is top of the charts? BPIFB1/LPLUNC1 localises to the bronchiolised epithelium in the honeycomb cysts in UIP}

Transcriptomic analyses are increasingly being used to define the molecular phenotype of complex diseases and may yet allow the identification of novel diagnostic and prognostic markers. Yang et $a l^{1}$ are to be congratulated for their meticulous application of this technology to the systematic study of gene signatures from patients with Idiopathic Pulmonary Fibrosis (IPF). This study, which appears to be the largest data set yet published, identifies two distinct gene signatures with high expression of 'cilium-associated genes' being shown to be associated with cases that exhibit microscopic honeycombing, which is commonly observed in, but not specific to, 'Usual Interstitial Pneumonia' (UIP), the pathological pattern of remodelling seen in IPF. This metaplastic bronchial type epithelium within the honeycomb spaces has recently been the focus of histological studies that have shown the strong expression of the gel forming mucin, MUC5B. ${ }^{2} 3$

This new expression signature contains many genes previously shown to be upregulated in IPF/UIP, including MUC5B, as well as many that appear to be novel, offering the promise of potential disease biomarkers. The online supplementary data show that the most differentially expressed gene within this data set is BPIFB1/LPLUNC1 (identified as the rather anonymous, C20orf114), a member of a poorly characterised gene family normally found in airway submucosal glands and within a population of airway goblet cells. ${ }^{4}$ We have previously highlighted how members of this gene family are among the top hits in many pulmonary array studies. ${ }^{5}$ Analysis of additional published data sets from IPF/UIP (see online supplementary table S1) confirms that elevated expression of BPIFB1 is strongly associated with IPF. We used immunohistochemistry to localise BPIFB1 in 10 cases of UIP (figure 1). In distal airways (associated with clear smooth muscle bands), the protein is localised to goblet cells that line the airways. In addition, the protein also stains the smaller airways, as well as the luminal contents. Similar staining patterns are seen in sections stained with MUC5B. In this location, the staining is not dissimilar to that seen in chronic obstructive pulmonary disease. In the smaller airways, BPIFB1 and MUC5B also exhibit overlapping localisation, whereas staining with the other common gel-forming mucin MUC5AC is more limited. In the honeycomb cysts, BPIFB1 clearly localises to the surface epithelial goblet cells that produce MUC5B (also see online supplementary figure S1), whereas these are negative for BPIFA1 (a serous cell marker) and SCGB1A1 (a Clara cell marker). The same pattern of staining was not seen in hypersensitivity pneumonitis (10 cases), a condition in which cystic bronchiolisation is also observed (see online supplementary figure S2) or bronchiolisation associated with lung cancer, suggesting a degree of specificity for UIP-associated bronchiolised epithelium. Our protein data extend the expression data from Yang et $a l^{1}$ and suggest that BPIFB1 may be a useful marker for the development of the honeycomb cysts that are characteristic of UIP.

Colin D Bingle, ${ }^{1}$ Bianca Araujo, ${ }^{1}$ William A Wallace, ${ }^{2}$ Nik Hirani, ${ }^{3}$ Lynne Bingle ${ }^{4}$

${ }^{1}$ Department of Infection and Immunity, University of Sheffield, Sheffield, UK

${ }^{2}$ Department of Pathology, University of Edinburgh, Edinburgh, UK

${ }^{3}$ MRC Centre for Inflammation Research, University of Edinburgh, Edinburgh, UK

${ }^{4}$ School of Clinical Dentistry, University of Sheffield, Sheffield, UK

Correspondence to Dr Colin Bingle, Academic Unit of Respiratory Medicine, Department of Infection and 


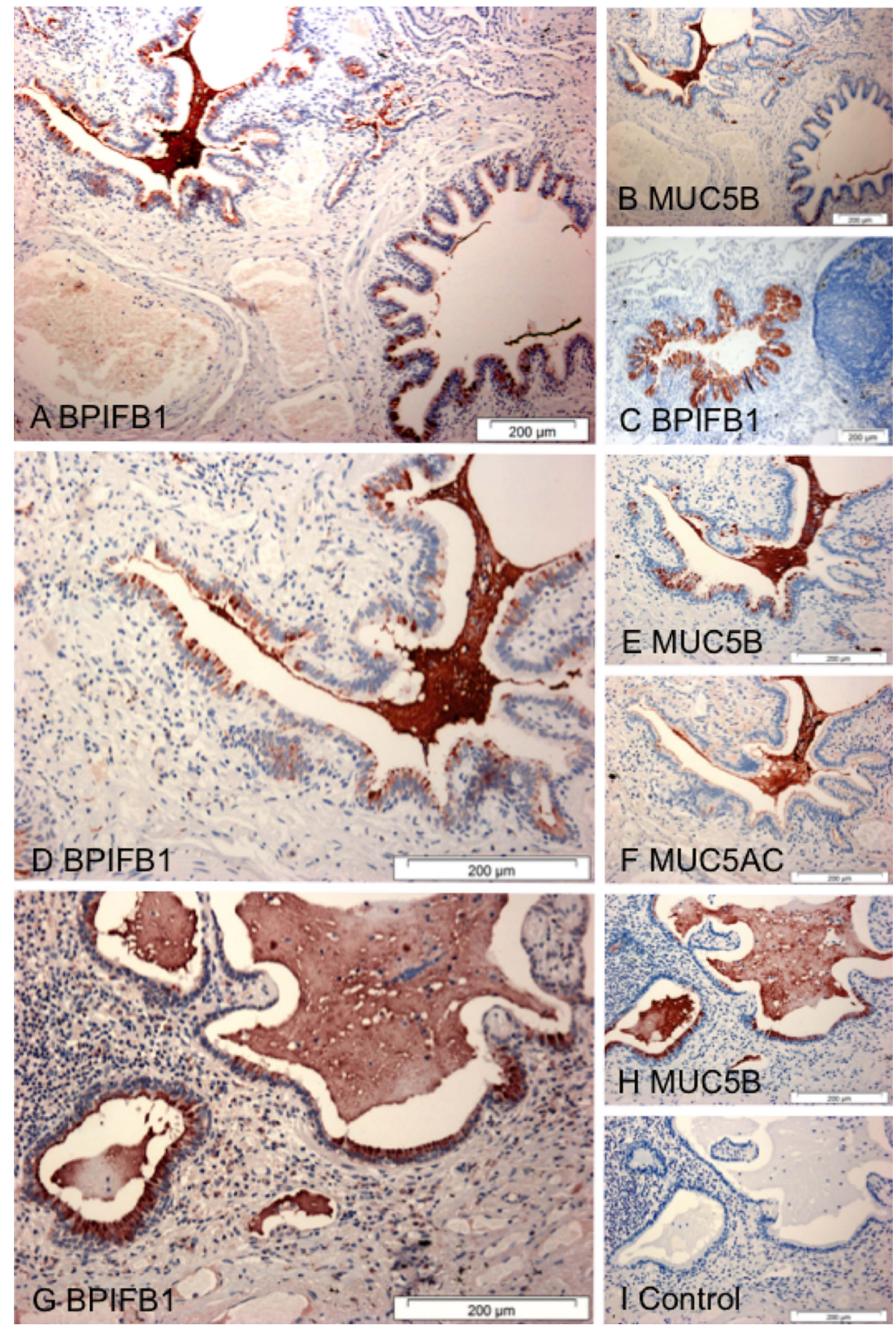

Figure 1 BPIFB1 colocalises with the MUC5B in the Bronchiolized epithelium in Usual Interstitial Pneumonitis (UIP). Immunohistochemistry for BPIFB1 (A, C, D, G), MUC5B $(B, E, H)$ and MUC5AC (F) was performed as described ${ }^{4}$ using sections from patients with UIP $(A, B, D-I)$ or from Chronic obstructive pulmonary disease (C). A no antibody control is included (I). Scale bars are present on each individual panel.
Immunity, University of Sheffield, Sheffield S10 2JF, UK; c.d.bingle@sheffield.ac.uk

- Additional material is published online only. To view please visit the journal online (http://dx.doi.org/10. 1136/thoraxjnl-2013-204179).

Contributors $C D B$ and $L B$ conceived the study. $B A$ and $\mathrm{LB}$ undertook the $\mathrm{IHC}$ work. CDB, BA and $\mathrm{LB}$ took the images. $\mathrm{NH}$ and WAW were responsible for patient identification, diagnosis and provided the samples. WAW provided pathological input.

Funding European Respiratory Society (grant number MC1562-2010).

Competing interests BA was supported by an ERS Long Term Research Fellowship.

Ethics approval South Sheffield Research Ethics Committee.

Provenance and peer review Not commissioned; internally peer reviewed.

To cite Bingle $C D$, Araujo B, Wallace W A, et al. Thorax 2013;68:1167-1168.

Received 11 July 2013

Revised 16 August 2013

Accepted 22 August 2013

Published Online First 7 September 2013

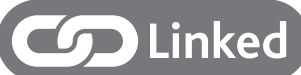

- http://dx.doi.org/10.1136/thoraxjnl-2012-202943

Thorax 2013:68:1167-1168.

doi:10.1136/thoraxjnl-2013-204179

\section{REFERENCES}

1 Yang IV, Coldren CD, Leach SM, et al. Expression of cilium-associated genes defines novel molecular subtypes of idiopathic pulmonary fibrosis. Thorax 2013;68:1114-21.

2 Plantier L, Crestani B, Wert SE, et al. Ectopic respiratory epithelial cell differentiation in bronchiolised distal airspaces in idiopathic pulmonary fibrosis. Thorax 2011;66:651-7.

3 Seibold MA, Smith RW, Urbanek C, et al. The idiopathic pulmonary fibrosis honeycomb cyst contains a mucocilary pseudostratified epithelium. PLoS One 2013;8:e58658.

4 Bingle CD, Wilson $\mathrm{K}$, Lunn $\mathrm{H}$, et al. Human LPLUNC1 is a secreted product of goblet cells and minor glands of the respiratory and upper aerodigestive tracts. Histochem Cell Biol 2010;133:505-15.

5 Barnes FA, Bingle L, Bingle CD. Pulmonary Genomics, Proteomics, and PLUNCs. Am J Respir Cell Mol Biol 2008;38:377-9. 